EchoGéo

ECHOGEO Sur le Vif $\mid 2008$

\title{
La junte militaire birmane confrontée au Cyclone Nargis
}

\section{Marion Sabrié}

\section{(2) OpenEdition}

Journals

Édition électronique

URL : http://journals.openedition.org/echogeo/4572

DOI : 10.4000/echogeo.4572

ISSN : 1963-1197

Éditeur

Pôle de recherche pour l'organisation et la diffusion de l'information géographique (CNRS UMR 8586)

\section{Référence électronique}

Marion Sabrié, "La junte militaire birmane confrontée au Cyclone Nargis », EchoGéo [En ligne], Sur le Vif, mis en ligne le 03 juin 2008, consulté le 20 avril 2019. URL : http://journals.openedition.org/ echogeo/4572 ; DOI : 10.4000/echogeo.4572

Ce document a été généré automatiquement le 20 avril 2019.

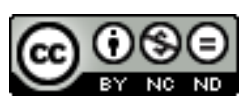

EchoGéo est mis à disposition selon les termes de la licence Creative Commons Attribution - Pas d'Utilisation Commerciale - Pas de Modification 4.0 International 


\title{
La junte militaire birmane confrontée au Cyclone Nargis
}

\author{
Marion Sabrié
}

1 L'Union de Birmanie, après avoir été oubliée par l'actualité depuis les manifestations de septembre et d'octobre 2007, a brièvement, de nouveau, fait la une des journaux: " Parodie d'ouverture diplomatique ? ", « Un génocide par omission? ", « Désastre naturel, catastrophe humanitaire », etc. En effet, le pays doit la recrudescence de sa médiatisation à deux évènements: le Cyclone Nargis de catégorie 4 qui a touché les Divisions de Rangoun, de l'Irrawaddy et de Bago (Pegu), ainsi que les Etats Môn et Karen les 2 et 3 mai 2008 et le référendum sur la Constitution birmane qui s'est déroulé le 10 mai 2008. Le " Cyclone Nargis », ainsi nommé par le service météorologique indien ou « Cyclone tropical 01B » selon le Joint Typhoon Warning Center américain (JTWC) a été qualifié par JeanFrancois Riffaut, porte-parole de la Croix-Rouge, de « désastre le plus important » depuis le Tsunami du 26 décembre 2004 qui avait fait 200000 morts en Inde du sud, au Sri Lanka, en Indonésie et dans le sud de la Thaïlande . 


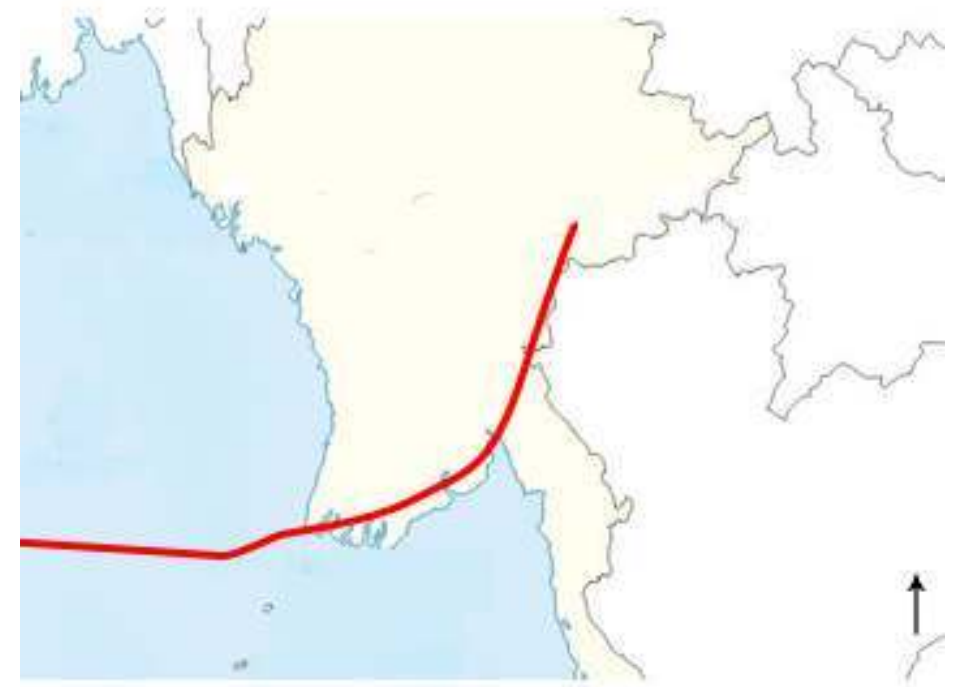

la trajectoire du cyclone nargis

2 L'Agence des Nations Unies pour la prévention des catastrophes a déploré l'absence d'alerte par le gouvernement birman, bien que le service météorologique indien (IMD) affirme avoir prévenu la Birmanie 48 heures à l'avance. Un premier bulletin aurait été envoyé dès la formation du cyclone le 26 avril. Selon le ministre birman des Affaires étrangères, le bilan au 5 mai 2008 était d'environ 15000 morts. Le 13 mai, il était de 32000 et 41000 disparus, tandis que les évaluations du Bureau des Nations Unies pour la coordination des affaires humanitaires (OCHA) oscillent entre 63300 et 101000 morts et 220000 disparus. Au 28 mai, le nombre de morts était évalué à 133600 . Certains diplomates avancèrent le chiffre de 100000 morts mettant en doute et attirant l'attention sur les épidémies à venir dues à l'inaccessibilité à l'eau potable (salinisation de l'eau, pollution des pluies et destruction partielle du système de distribution). Bilan encore plus redoutable lorsqu'on sait que le budget pour la santé s'élève à peine à $3 \%$ du budget global de l'Etat birman. Cette catastrophe a lieu au début de la mousson birmane, moment où la recrudescence des maladies, dont le paludisme, fait habituellement rage. La population sinistrée se chiffre entre un et deux millions. La tempête a inondé toute la zone littorale de la Division de l'Irrawaddy. Une vague de 3,5 mètres a submergé environ $5000 \mathrm{~km}^{2}$ selon Richard Horsey, le porte-parole de l'ONU à Bangkok. Certaines villes et villages ont été dévastés à plus de $80 \%$. 


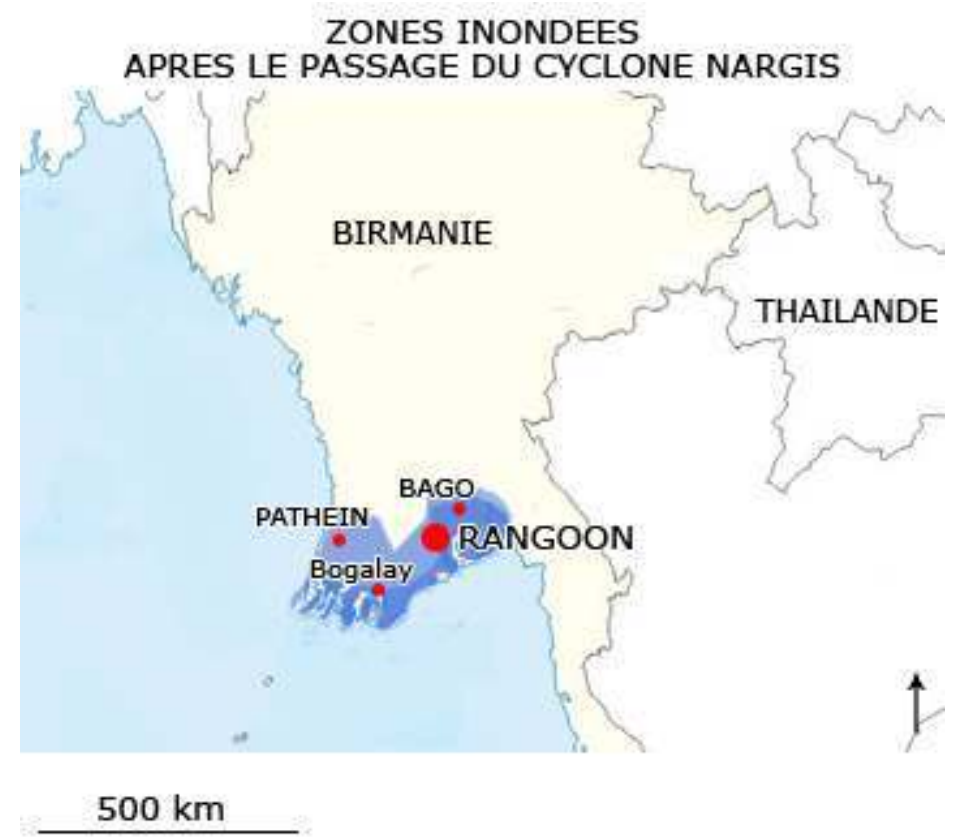

Les divisions de Rangoun et de l'Irrawaddy dont Rangoun et Pathein sont les capitales ont été majoritairement touchées.

La catastrophe naturelle a-t-elle véritablement des répercussions sur la situation politique de la Birmanie? A l'échelle nationale, l'impact semble moindre car la junte n'est pas prête à la moindre concession et est toujours en position de force face au peuple. Sur la scène internationale, la tenue du référendum a porté au comble l'indignation générale et la colère préexistantes à cet évènement. Le référendum a malgré tout été maintenu le 10 mai, excepté dans les régions sinistrées où le régime a concédé son report au 24 mai. Premier référendum depuis les élections législatives de mai 1990, il s'est soldé par un "succès" dont la junte s'est aussitôt félicitée. Le journal de propagande étatique, le New Light of Myanmar, rapporte que les Birmans « ont massivement participé au référendum ». Du point de vue du pouvoir, le référendum constitutionnel a encore un sens, même après une catastrophe de l'ampleur du cyclone Nargis: il ne semble pas y avoir d'avant et d'après catastrophe. D'un point de vue humain, ce référendum semble totalement déplacé lorsque le peuple meurtri pense avant tout à sa survie. Le référendum constitutionnel est une réponse à la demande internationale répétée d'opter pour "une voie vers la démocratisation" de l'appareil dictatorial birman. Certes le référendum semble user de moyens démocratiques, mais c'est une façon d'entériner l'accession illégitime de la junte au pouvoir et de lui donner une justification et une assise constitutionnelles « validées par le peuple ». A l'instar de nombreux gouvernements et organisations internationales, dont l'Union Européenne par la voix de son envoyé spécial, l'Italien Piero Fassino, Aung San Suu Kyi - principale figure de l'opposition birmane - qualifie le référendum d'insulte au peuple, resté exsangue par le passage du Cyclone.

\section{Un scrutin sous haute surveillance}

Le corps électoral est évalué à 30 millions. Les médias dénoncent la pression exercée par les autorités locales sur la population des villes comme des campagnes durant les quelques mois après l'annonce de la tenue du référendum et de possibles élections en 
2010. Ils se font désormais l'écho des fraudes électorales qui ont eu lieu. Le vote s'est déroulé en l'absence de toute Commission Internationale d'Observation, refusée par la junte militaire. Parmi la société civile birmane, il y a de nombreux informateurs à la solde du gouvernement. Ce sont parfois des activistes n'ayant pas eu le choix entre l'espionnage, la prison ou la mort, ou de simples quidams désignés par les organisations locales de contrôle de la population. A chaque niveau la société locale est surveillée, ce qui rend illusoire la possibilité du scrutin libre et démocratique. Le New Light of Myanmar titre qu' " approuver la Constitution étatique est un devoir national ». Le processus importe peu, c'est la fin qui compte: une confortation du pouvoir national aux yeux de la scène internationale. Le peuple ne connaît pas le texte constitutionnel et n'y a pas accès. Quant à l'opposition, il faut considérer d'une part, la traditionnelle opposition de la NLD, la Ligue Nationale pour la Démocratie, et de l'autre, les nouveaux activistes. La seule information véhiculée est le probable renforcement du pouvoir des militaires au gouvernement. Du point de vue majoritaire de la population locale, tout semble comme si le référendum était un référendum pour ou contre la dictature.

5 Malgré les efforts de l'opposition en Birmanie et sur la scène internationale en faveur du non, le oui l'a emporté. Bien que l'opposition meurtrie par la répression des manifestations de 1988 et celles de 2007 subsiste toujours, un mouvement massif de contestation politique, d'une ampleur similaire au mouvement mené par les bonzes en 2007,semble peu probable. Avant le cyclone Nargis, la population birmane semblait découragée et désemparée par les évènements de septembre et d'octobre 2007. Chaque famille birmane a un proche ou une connaissance ayant été victime de la répression: soit arrêté, torturé ou exécuté (voire les trois). Ces évènements ont montré que la junte militaire n'hésite pas à réprimer dans le sang tout mouvement contestataire même si elle doit user de sa force contre le "Sangha ", la communauté des moines bouddhistes incontestablement respectée par la population birmane dont le Bouddhisme constitue une des caractéristiques majeures de l'identité.

La junte militaire semble prête à tout mettre en œuvre pour consolider son pouvoir et la " voie vers la démocratisation » souhaitée par la NLD, le parti politique d'opposition, et par la scène internationale ne semble possible qu'en passant par un bain de sang. Les Birmans sont désormais convaincus que la voie pacifique est illusoire. Ils craignent une nouvelle répression car les mouvements contestataires désorganisés ne possèdent pas, à l'heure actuelle, assez d'armes en comparaison avec l'armée birmane, le "Tatmadaw ", qui représente $40 \%$ du budget de l'Etat birman. Un des problèmes majeurs d'un mouvement de contestation politique demeure la désunion des mouvements d'opposition et de rébellion, birmans ou des minorités ethniques. Ce problème subsiste depuis l'échec du fédéralisme dans les débuts de la Birmanie indépendante. Un des problèmes révélés au passage par le cyclone Nargis est celui d'un pays à deux vitesses: les régions touchées par le Cyclone, c'est-à-dire les divisions de l'Irrawaddy et de Rangoun, d'une part, et celles épargnées, d'autre part. Les zones sinistrées par ce que les Nations Unies ont qualifié de " désastre majeur " se soucient de leur reconstruction, du " retour à la normale " des facilités telles que l'eau et l'électricité. (Il faut cependant savoir qu'en temps normal aucun des quartiers de Rangoun, l'ancienne capitale et pôle économique majeur du pays, n'a d'électricité $24 \mathrm{~h} / 24$. La majeure partie des citadins est habituée à 10/12 heures d'électricité par jour par tranche de 5/6 heures exceptées les familles les plus aisées et de nombreuses entreprises qui peuvent se permettre l'achat d'un générateur. Après le cyclone, face à la hausse exorbitante du prix du pétrole, le ravitaillement apparaît un luxe 
réservé à une minorité très privilégiée. Le ravitaillement en eau des habitations est entièrement dépendant de l'électricité. Les pompes à eau ne fonctionnent que durant les plages horaires où il y a de l'électricité. Ce système, déjà défaillant et insuffisant en temps normal, désormais anéanti par le passage du cyclone, devrait être rétabli dans les deux mois à venir au minimum.) Même si parmi les Birmans, une colère grandissante à l'égard du gouvernement se fait sentir, notamment depuis la répression des moines, figures hautement vénérées par la population civile, c'est une colère doublée d'une grande lassitude : les nouvelles générations n'ont vécu que sous un régime dictatorial instauré depuis 1962. Colère, mais pas encore révolte. En effet, une majeure partie des birmans pensent selon les préceptes bouddhiques que la destinée de la Birmanie, dictature militaire et cyclone, doit être ainsi et que leurs actions de lutte contre des phénomènes " divins " sont vaines. "Sur le chemin de la démocratisation, la voie semble encore bien longue pour une société aussi militarisée que la Birmanie " (voir l'article de l'auteur, "Birmanie : vers une démocratisation ou un renforcement de l'appareil dictatorial?", EchoGéo, Sur le vif, décembre 2007.) Le référendum et la possibilité de la tenue d'élections en 2010 apparaissent comme une "Opération Séduction » de la junte vis-à-vis de la communauté internationale qui n'est pas dupe. Concrètement, le résultat a été " truqué " à l'avance. Sur la scène internationale, la Birmanie semble encore plus isolée qu'auparavant, bien que la Chine ait réitéré son soutien en octroyant une aide financière de 250000 dollars et surtout en s'opposant au vote de la résolution proposée par la France aux Nations-Unies pour imposer une aide humanitaire au gouvernement birman. La Chine a argué que les questions humanitaires n'étaient pas du ressort du Conseil de Sécurité des Nations Unies. Les intérêts de la Chine sont avant tout politiques et économiques. Néanmoins, le 24 mai, Yang Jiechi, le chef de la diplomatie chinoise, conscient de l'ampleur de la catastrophe, a déclaré que la communauté internationale devait continuer à aider la Birmanie après le cyclone. Dix jours après le passage de Nargis en Birmanie, la région du Sichuan, sud-ouest de la Chine, a été touchée par un tremblement de terre. Pékin a eu une première réaction similaire à celle de la junte : accepter l'aide internationale mais pas les équipes. Les médias semblaient moins troublés. La Chine est finalement revenue sur sa décision et a opté pour la transparence : la communauté internationale a pu conjointement avec les troupes participer à l'aide d'urgence, les soins médicaux et à la mise en place de la reconstruction. Cependant toute aide et le moyen de la mettre en oeuvre a exigé et exige encore des consultations bilatérales. C'est une accessibilité et une ouverture similaires qu'espère encore la communauté internationale à l'égard de la catastrophe en Birmanie. La situation politique birmane sur le plan national et international apparait dans la même impasse qu'avant la catastrophe naturelle. La junte n'a fait que se discréditer davantage aux yeux de son peuple et du monde. Il y a eu certains cas de désertion de soldats pour rejoindre leurs familles en péril, mais ni les évènements de 2007 ni les problèmes dans la gestion de la situation actuelle birmane n'ont entraîné de tension majeure au sein du régime. Malgré de nouvelles sanctions par la communauté internationale et la catastrophe humaine, la junte militaire ne paraît pas ébranlée et continue à accepter l'aide matérielle. Le 23 mai, Senior Général Than Shwe promettait à Ban Ki-moon, le Secrétaire Général des Nations Unies, lors de sa visite à Rangoun, une ouverture du pays aux travailleurs internationaux sans considération de leur nationalité. Bien qu'elle ait donc tenu le discours opposé, la junte minimise encore l'entrée de travailleurs humanitaires étrangers sur son territoire. Le 24 mai, 52 pays et 24 organisations ont participé à la Conférence des donateurs qui s'est tenue à Rangoun et où la junte a demandé 7 milliards d'euros pour la reconstruction 
de la zone. Pour les organisations internationales, il s'agissait davantage d'éliminer les différents obstacles à l'acheminement de l'aide aux populations. Au 30 mai, seul un nombre restreint de travailleurs médicaux de pays de l'ASEAN, à laquelle la Birmanie appartient depuis 1997, ont facilement obtenu leurs visas. Le problème reste donc l'obtention des visas mais surtout les autorisations pour accéder à la zone de distribution de l'aide. Le 28 mai, toute la zone du Delta de l'Irrawaddy n'est toujours pas accessible et les initiatives birmanes privées sont majoritairement contrôlées et/ou bloquées par les militaires sur place. A la périphérie de Rangoun, depuis le 24 mai, des barrages militaires ont cependant disparu. La majeure partie des ONG sur place s'accorde pour affirmer que seulement $10 \%$ à $20 \%$ de l'aide arrive à destination des sinistrés dans le besoin. Ce sont la plupart du temps les mêmes villages où l'aide peut se rendre. Les mois à venir dévoileront davantage l'ampleur de la catastrophe humaine.

Quelques images après le passage du cyclone

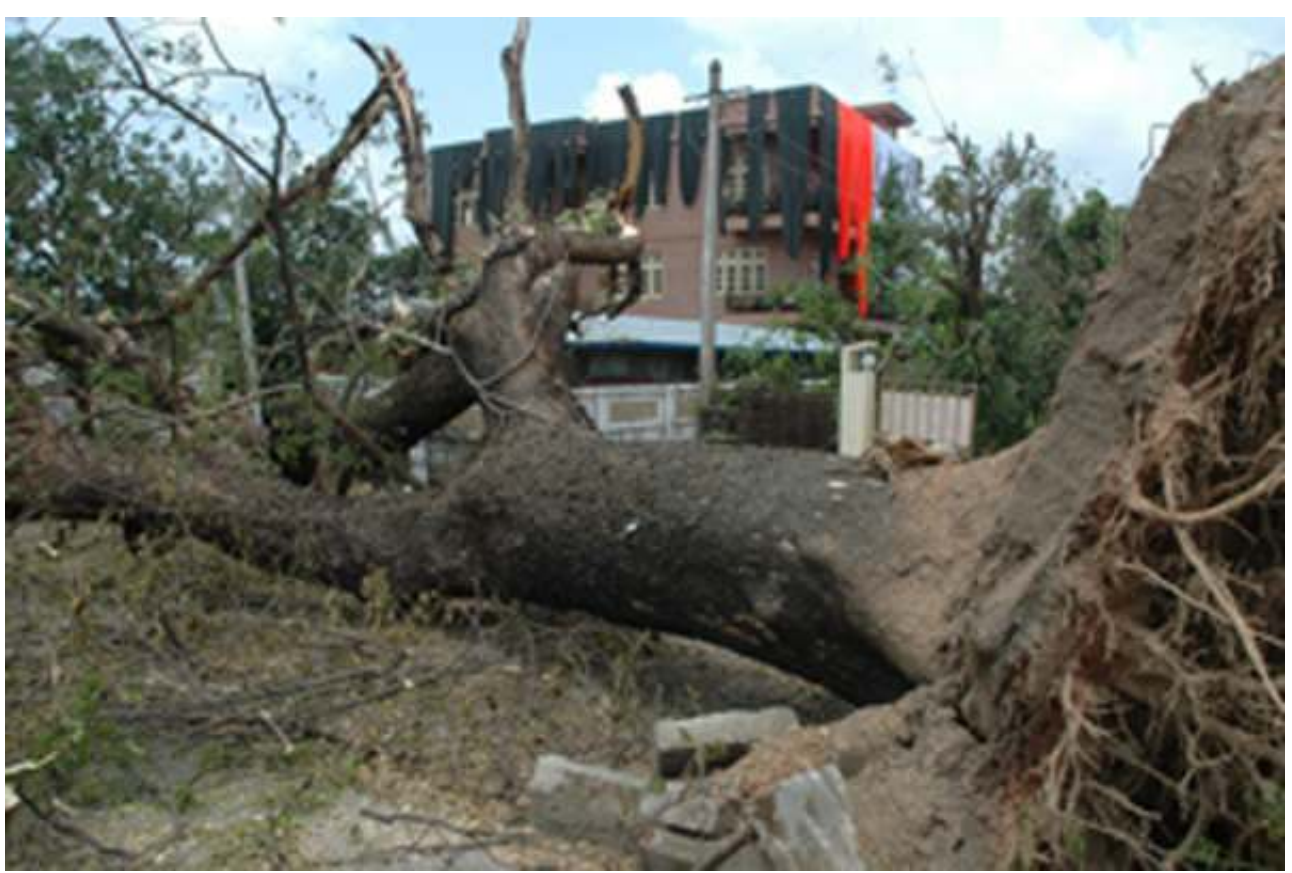



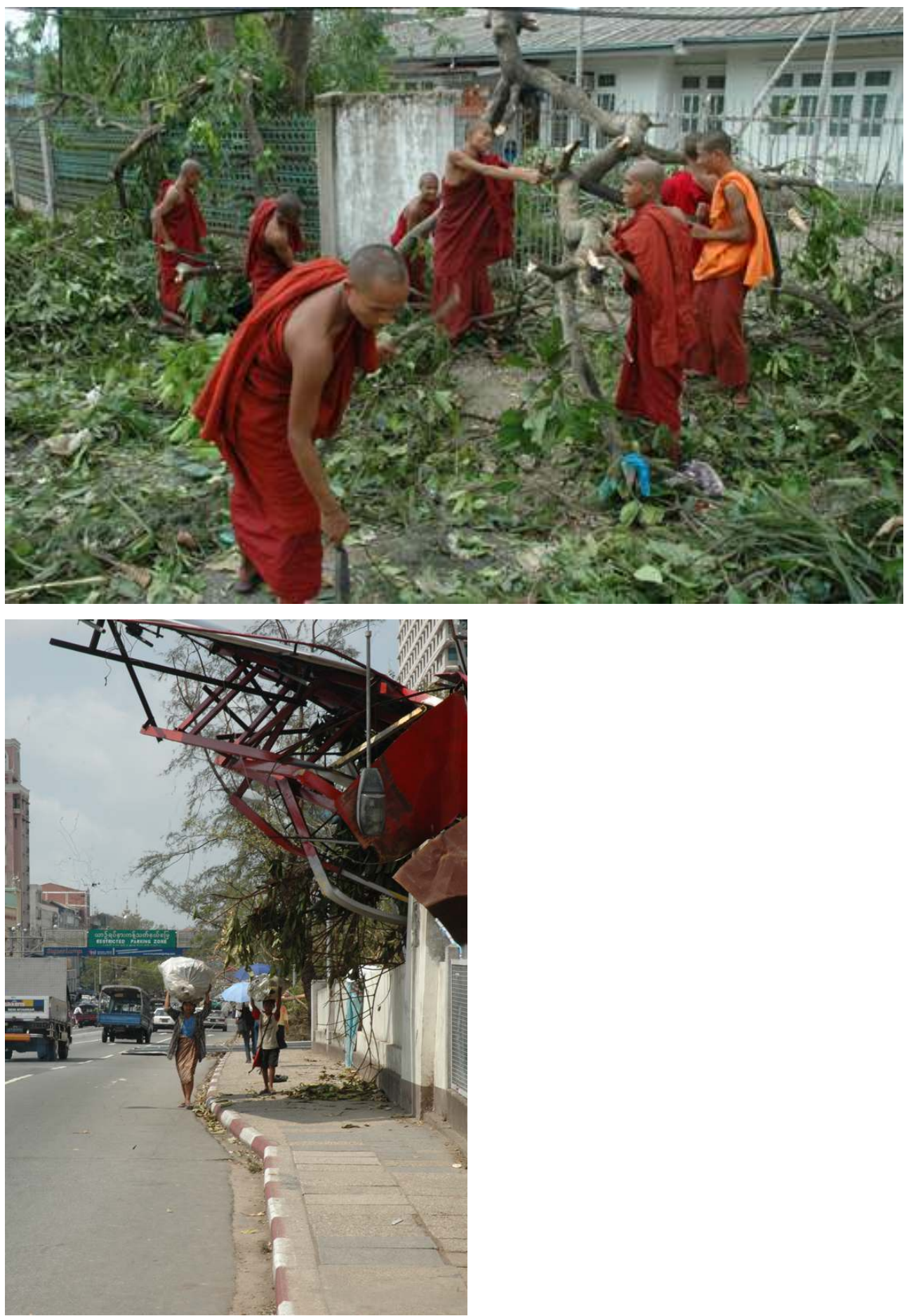


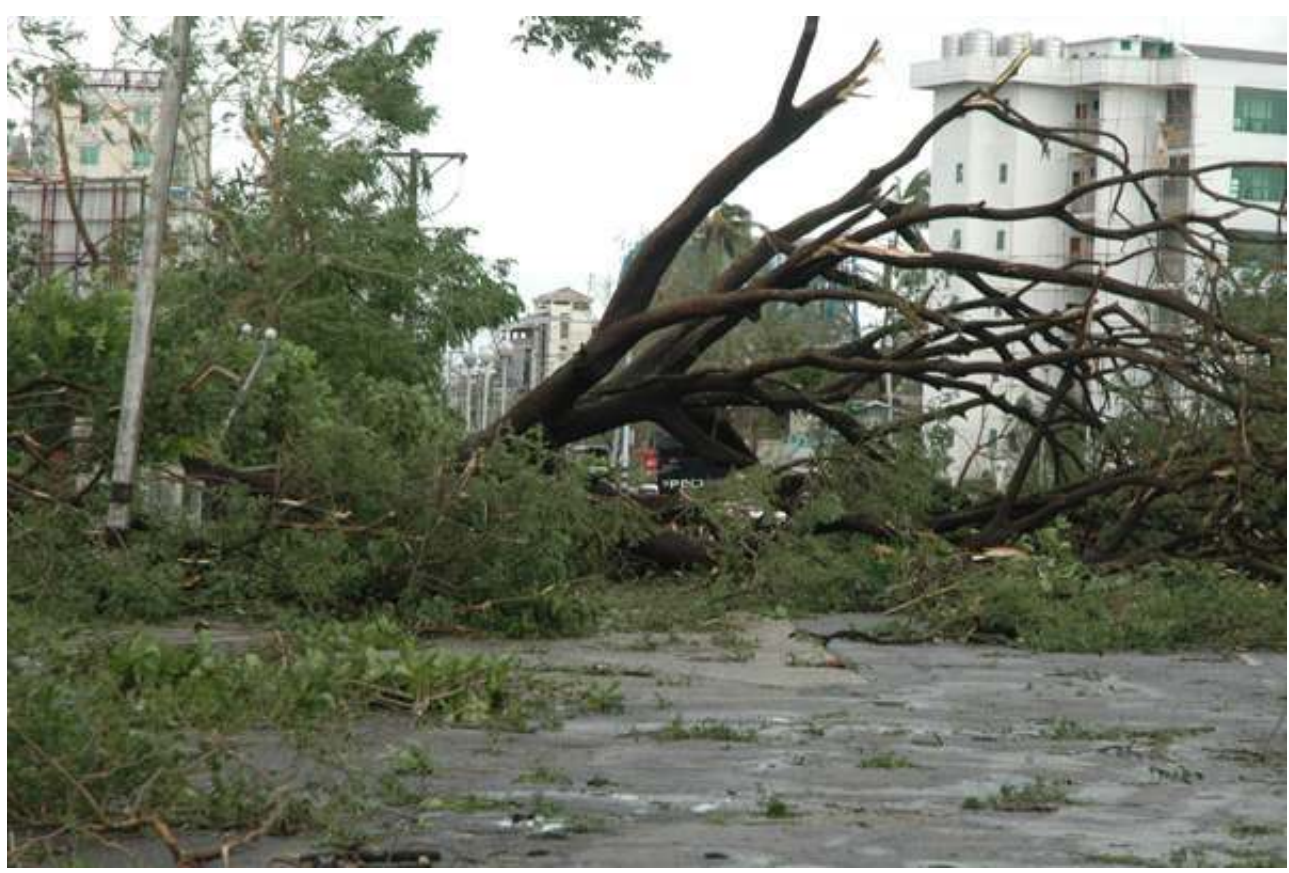

\section{RÉSUMÉS}

Les 2 et 3 mai 2008, le cyclone Nargis s'abat sur les Divisions de l'Irrawaddy et de Rangoun faisant 32000 morts, 41000 disparus et 2 millions de sinistrés. Face à l'inertie de la junte et à son refus de laisser entrer l'aide humanitaire, une «nouvelle catastrophe dans la catastrophe» se joue. Malgré l'ampleur du désastre, la junte militaire maintient le référendum constitutionnel une semaine plus tard.

On the $2^{\text {nd }}$ and the $3^{\text {rd }}$ of May 2008, the Cyclonic Storm Nargis made landfall on the Ayeyarwady Division and on Rangoun Division causing 32000 deaths, 41000 missing people and 2 million of homeless people. The military junta inactivity and its refusal to accept humanitarian aid lead to a "new tragedy in the disaster". Despite this disaster, the junta maintained the referendum about Constitution one week after.

\section{AUTEUR}

\section{MARION SABRIÉ}

Marion Sabrié (marion.sabrie@no-log.org) est en thèse à l'Université Paris-Sorbonne sous la direction d'Olivier Sevin. Elle est actuellement sur son terrain, la Birmanie, dont elle maîtrise la langue. 